\title{
Microglial targets for effective therapies of Alzheimer's disease
}

\author{
Klegeris $\mathbf{A}^{*}$ \\ Department of Biology, University of British Columbia Okanagan Campus, Kelowna, British Columbia, V1V 1V7, Canada
}

\section{Introduction}

Alzheimer's disease (AD) is the most common cause of dementia. The main pathological markers of this disease include amyloid plaques and neurofibrillary tangles. Research conducted over the last forty years has identified amyloid beta peptide $(\mathrm{A} \beta)$ as the main constituent of the plaques and characterized the abnormal metabolism of the amyloid precursor protein that leads to aggregation and deposition of these peptides in $\mathrm{AD}$ brains [1]. The intense preclinical and clinical research effort aimed at modulating $\mathrm{A} \beta$ metabolism as the means of slowing down or arresting $\mathrm{AD}$ has not, unfortunately, led to effective therapeutic agents or strategies, prompting questions about the suggested central or unique role of $\mathrm{A} \beta$ in $\mathrm{AD}$ [2]. Worldwide, more than 46 million people are affected by dementia. If no effective treatment is identified, this number is expected to double every 20 years. This illustrates the urgent need for identification and testing of alternative molecular and cellular targets that would allow effective modulation of $\mathrm{AD}$ pathological processes and clinical outcomes [1]. In the sections below, potential microglia-related targets are identified that could be used to design novel therapeutic agents for $\mathrm{AD}$.

\section{Microglia physiology}

Microglia represent an ontogenetically distinct population of mononuclear phagocytes, which originate from yolk sac progenitors at the early stages of embryogenesis; therefore, they are considered the resident macrophages of the central nervous system (CNS) $[3,4]$. Microglia participate in a range of physiological processes during brain development and maturation. For example, they secrete brain-derived neurotrophic factor (BDNF), insulin-like growth factor-1 (IGF-1), and several other neurotrophic factors that support neurogenesis. Since many unwanted cells have to be removed during neurodevelopment, microglia are essential for elimination of such cells through phagocytosis $[5,6]$. Microglia also closely associate with the developing CNS vasculature and genetic ablation of these cells leads to a sparser vascular network, indicating the supportive role of microglia in angiogenesis. To promote angiogenic sprouts, thus facilitating the formation of the vascular network, microglia secrete factors different from vascular endothelial growth factor-A (VEGF-A) [7].

In the mature CNS, microglial growth factors are essential for the formation of new synapses, for example, as part of the hippocampal neurogenesis, but microglia also participate in synaptic pruning by phagocytosing inactive synapses that have been labeled with the complement proteins $\mathrm{C} 1 \mathrm{q}$ and $\mathrm{C} 3[8,9]$. Under physiological conditions, most microglia are characterized by highly branched processes displaying ramified morphology. Due to the observed tilelike network that microglia form covering the CNS parenchyma, it was initially hypothesized that these cells did not touch or overlap with each other. Recently, two-photon excitation microscopy has been used to demonstrate that these cells, initially believed to be in resting state, in fact are actively sampling CNS environment. They are touching neighbouring cells and blood vessels by extending and retracting their thin, branched processes; therefore, microglia are constantly surveying brain parenchyma and are able to react and move quickly to sites of injury or invasion by pathogens [10-12].

\section{Microglia and the neuroinflammatory hypothesis of Alzheimer's disease}

Identification of reactive microglia in the post-mortem brain tissues of $\mathrm{AD}$ patients served as the initial evidence of the neuroimmune response being launched in the brain areas heavily invaded by plaques and tangles, the main histological markers of $\mathrm{AD}$ neuropathology. Up until then, AD was considered to affect neurons selectively. These initial studies demonstrated that the hippocampal and cortical structures affected by the disease were infiltrated by reactive microglia as well as astrocytes, which are another type of the non-neuronal glia of the brain. Reactive microglia were identified as having swollen, amoeboid shape. They were phagocytic, and were overexpressing the major histocompatibility complex (MHC) class II human leukocyte antigen (HLA)-DR isotype proteins on their surface as revealed by the immunohistochemistry techniques $[13,14]$.

The neuroinflammatory hypothesis of $\mathrm{AD}$, which states that gliadriven immune processes contribute to the onset and progression of this disease, is now supported by several other lines of evidence: 1) The reactive phenotypes of microglia and astrocytes in $\mathrm{AD}$ brains have been confirmed by identifying a range of other morphological and functional parameters [15]; 2) Biochemical and molecular biology studies have demonstrated upregulated inflammatory cytokines, chemokines, and complement in brain tissue affected by $\mathrm{AD}$ as markers of neuroimmune activation [16]; 3) More than a dozen epidemiological studies have identified reduced risk of $\mathrm{AD}$ in chronic users of anti-inflammatory drugs [17]; 4) Positron emission tomography (PET) techniques using a specific ligand for the mitochondrial translocator protein (TSPO, also known as the peripheral benzodiazepine binding site), which labels

${ }^{*}$ Correspondence to: Andis Klegeris, Department of Biology, 3187 University Way, University of British Columbia Okanagan Campus, Kelowna, British Columbia, V1V 1V7, Canada, Tel: 1-250-8079557, E-mail: andis.klegeris@ubc.ca

Key words: amyloid beta protein, DAMPs, drug targets, glial cells, neuroinflammation, neuroprotection

Received: March 01, 2020; Accepted: March 11, 2020; Published: March 17, 2020 
reactive microglia, show involvement of these cells at the early stages of $\mathrm{AD}$ pathogenesis [18]; 5) Upregulated inflammatory markers and glial activation are observed in the animal models of $\mathrm{AD}$; however, it is important to note that there are significant phenotypic and functional differences between reactive microglia, as well as astrocytes, from human $\mathrm{AD}$ tissues and AD mouse model brains [19,20]; 6) Genomewide association studies have revealed several inflammation-related genes as late-onset $\mathrm{AD}$ risk factors with low to moderate effect sizes. Examples of these genes, which are mainly expressed by microglia in $\mathrm{AD}$ brains, include triggering receptor expressed on myeloid cells 2 (TREM2); cluster of differentiation (CD) 33 (SIGLEC-3, sialic acidbinding Ig-like lectin 3); complement receptor 1 (CR1); phospholipase C gamma 2; and inositol polyphosphate-5-phosphatase [21,22].

\section{Pro-inflammatory activation of microglia in AD}

The initial reports of reactive microglia in $\mathrm{AD}$ demonstrated that these cells surrounded A $\beta$-containing senile plaques [13,14]. Since then, it has been shown by using cultured cells and animal models of $A D$ that microglia can be adversely activated by $\mathrm{A} \beta$, which leads to secretion of inflammatory mediators. Based on the previously described M1 and M2 activation states of peripheral macrophages [23], it has been proposed that $\mathrm{A} \beta$ can induce the so-called classical pro-inflammatory M1 phenotype of microglia. Such pro-inflammatory activation of microglia is also induced by established immune stimuli interferon (IFN) $-\gamma$ and lipopolysaccharide (LPS) [24]. In addition to the evidence collected by using isolated microglia cultures and $\mathrm{AD}$ animal models, a significant support to the proposed adverse contribution of microglia to the disease pathogenesis comes from clinical PET imaging studies showing strong correlation between reactive microglia and cognitive decline in $\mathrm{AD}$ patients [25]. Since the inflammatory mediators and cytotoxins released by M1 microglia could be at least partially responsible for the neuronal death in $\mathrm{AD}$, preventing or slowing down microglial transition from the "surveying" to M1 phenotype could be an effective strategy for attenuating $\mathrm{AD}$ progression $[10,26,27]$.

Several molecular targets have been proposed for achieving this goal. As a result of an intense research effort, more than ten different microglia receptors have been identified that can mediate their proinflammatory activation by $A \beta$. They include receptor for advanced glycation end products (RAGE), toll-like receptors (TLRs) 2 and 4, macrophage receptor with collagenous structure (MARCO), CD36, formyl peptide receptor 2 (FPR2), peroxisome proliferator-activated receptors (PPARs) $\gamma, \delta$, and $\alpha$, and liver X receptors (LXR) $\alpha$ and $\beta$. It has also been established that microglial activation by $A \beta$ depends on the polymerization state of this peptide with oligomeric soluble $A \beta$ being more pro-inflammatory than its insoluble fibrillar forms. Several distinct intracellular signaling molecules mediate the pro-inflammatory transformation of microglia including dual specificity phosphatase 2 (DUSP2), nuclear factor (NF) $\kappa$ B, and the three main mitogen-activated protein kinases (MAPKs): extracellular signal-regulated kinase (ERK), c-Jun terminal kinase/stress-activated protein kinase (JNK/SAPK), and p38 MAPK (for reviews see [28-31]). Antagonists of the microglia receptors that are activated by the soluble oligomeric $A \beta$ and inhibitors of key intracellular signaling pathways engaged by these receptors could be developed as therapeutic agents in AD.

Soluble $A \beta$ oligomers are not the only microglial stimulants present in the extracellular space of AD brain parenchyma. Several different damage-associated molecular patterns (DAMPs) have been shown to be released from damaged neurons and/or glial cells. For example, the non-histone DNA binding protein high-mobility group box 1 (HMGB1), normally located in the cell nucleus, is released passively from dying cells or actively secreted through several different pathways by stimulated cells [32-34]. Cytochrome $\mathrm{C}$ and mitochondrial transcription factor A (TFAM), which are mitochondrial proteins, can also be released into extracellular space and activate microglia [35-37]. All these proteins act like typical DAMPs by binding to the pattern-recognition receptors (PRRs) expressed by microglia inducing their pro-inflammatory activation. Therefore, microglial PRRs such as RAGE, nucleotide-binding oligomerization domain-like receptors (NLRs), TLR 2 and TLR 4, as well as the intracellular signaling pathways associated with these receptors, could be targeted to inhibit adverse activation of microglia [38-40].

Reactive microglia can cause neuronal damage directly by phagocytosing stressed, but live, neurons, and by stripping dendritic spines in complement-dependent manner [41,42]. Pro-inflammatory microglia also release a mixture of neurotoxins whose composition most likely depends on the nature and concentration of stimulating agents present in the extracellular space. Even though significant interspecies differences have been noted [6], several well-characterized neurotoxins are secreted by microglia from different species. They include reactive oxygen species (e.g., superoxide anion), excitatory amino acids (e.g., L-glutamate), neurotoxic metabolites (e.g., quinolinic acid), phospholipases (e.g., secreted phospholipase A2 group IIA), and proteases (e.g., cathepsin B, prolyl endopeptidase) capable of killing neurons directly (reviewed in [43-48]). Designing selective therapeutic agents that scavenge or neutralize some of the toxins (reactive oxygen species), block neuronal receptors interacting with the neurotoxins (L-glutamate), or inhibit their enzymatic activity (proteases and phospholipases) could be a valid strategy for lowering the impact of adverse M1 activation of microglia.

In addition to their direct neurotoxicity, reactive microglia contribute to neuronal injury indirectly. Increased secretion of chemokine (C-C motif) ligand 1 (CCL1), CCL20, interleukin (IL) $1 \beta$, IL-6, and tumor necrosis factor (TNF) $\alpha$ is an established characteristic of the classical M1 activation phenotype of microglia [49]. Once released by reactive microglia, the above cytokines and chemokines can recruit and activate surrounding surveying microglia and astrocytes in paracrine manner. Interestingly, astrocytes have been recently shown to become neurotoxic in response to IL-1 $\alpha, \mathrm{TNF} \alpha$, and complement $\mathrm{Clq}$ secreted by reactive microglia. Such a neurotoxic state of astrocytes has been termed A1 phenotype and cells displaying this novel phenotype have been identified in brains of $\mathrm{AD}$ patients [50]. Neutralizing microglial pro-inflammatory cytokines and chemokines, as well as blocking their corresponding receptors on microglia and astrocytes, should be considered as a means of slowing down neuroinflammatory cascades in AD. A recent trend in new drug discovery effort should be noted, which involves multi-target directed ligand (MTDL)-based strategies incorporating two or more pharmacophores into a single drug molecule. In the MTDL drugs designed for AD, one or more of the pharmacophores could be directed at microglia-specific targets such as cytotoxic proteases, cell surface receptors, or intracellular signaling molecules [31].

\section{Anti-inflammatory activation of microglia in AD}

In $\mathrm{AD}$ brains, surveying microglia can transition not only to the M1 pro-inflammatory activation state but also to the alternative M2 anti-inflammatory/resolution activation state. The M2 phenotype can be induced by IL-4, IL-10, and IL-13, and is characterized by secretion of several anti-inflammatory cytokines including IL-10, IL-1 receptor 
antagonist (IL-1RA), and transforming growth factor (TGF- $\beta$ ). Lists of the secreted and cell-surface markers of the M1/M2 microglia phenotypes are partially overlapping and have been steadily growing. Such in-depth studies of reactive microglia have led to identification of new functional states including several subtypes of M2 activation [24,49,51]. It is important to note that both the M1 and M2 microglia have been detected not only in the brains of $\mathrm{AD}$ model mice but also in human post-mortem AD brain tissues [51,52]. Increasing evidence also supports existence of microglial activation states that do not align well with the M1/M2 polarization axis [53]; for example, both human and $\mathrm{AD}$ mouse model brains have been shown to possess a distinct population of disease-associated microglia (DAM), which accumulate around amyloid plaques [54].

Microglia have been shown to switch from M2 to M1 phenotype as $\mathrm{AD}$ pathology progresses in a double-transgenic mouse model [55]. While the dominance of $\mathrm{M} 1$ microglia during late $\mathrm{AD}$ is consistent with their adverse effects on neuronal survival, M2 activation of microglia at the early disease stages indicates these cells could have upregulated protective functions aimed at restoring brain homeostasis. It has been proposed that M2 microglia, as well as possibly DAM, are protective by phagocytosing $A \beta$ deposits and degrading extracellular $A \beta$ by secreting several different proteases, in addition to secreting anti-inflammatory cytokines $[49,54,56]$. While some of the microglia receptors, such as CD36, TLR2, and TLR4 have been associated with both $A \beta$ phagocytosis and pro-inflammatory adverse activation, several receptors contribute to $A \beta$ phagocytosis by microglia with relative selectivity; they include scavenger receptor A-1 (SCARA-1) and chemokine-like receptor 1 (CMKLR1) [30]. Thus, therapeutic agents that facilitate transition of microglia to M2 phenotype either from the surveying or M1 state could be beneficial in $\mathrm{AD}$ due to upregulated $\mathrm{A} \beta$ clearance capacity of microglia and secretion of anti-inflammatory cytokines.

The functional state of microglia can be regulated by a diverse set of soluble molecules as well as through direct contact with other cells and insoluble structures such as amyloid plaques [5,51]. In addition to DAMPs discussed above, there is an emerging group of molecules termed resolution-associated molecular patterns (RAMPs), which inhibit the secretion of pro-inflammatory cytokines and cytotoxins by immune cells [57]. Thus far, RAMPs have been mainly studied in the context of peripheral diseases; however, evidence is accumulating that some of these molecules reduce adverse activation of microglia and facilitate removal of $A \beta$ aggregates. For example, galectin- 1 has been shown to deactivate M1 microglia [58], binding immunoglobulin protein (BiP, GRP78) stimulates A $\beta$ clearance by microglia [59], and extracellular cardiolipin upregulates microglial phagocytosis and expression of neurotrophic factors, but decreases their secretion of inflammatory mediators and cytotoxins [60]. Furthermore, disease symptoms can be reduced in $\mathrm{AD}$ model mice by overexpressing heatshock protein 27 (HSP27) [61] or administering cardiolipin-containing liposomes [62] illustrating that some of the RAMPs could have a potential as therapeutic agents in $\mathrm{AD}$.

\section{Conclusion}

The contribution of neuroinflammation to $\mathrm{AD}$ pathogenesis as well as the central role of microglia in neuroimmune processes are becoming increasingly recognized. Reactive microglia can play both beneficial and detrimental roles in $\mathrm{AD}$; therefore, future studies should identify therapeutic agents and strategies that can selectively downregulate or mitigate harmful activity of microglia, including their secretion of pro-inflammatory cytokines and neurotoxins, while preserving or even upregulating their beneficial functions such as phagocytosis and removal of $A \beta$. Development of such therapeutic agents and strategies is challenging due to the shifting balance between M1/M2 microglia in $\mathrm{AD}$ progression, the significant overlap of receptors and signaling molecules regulating the beneficial and the harmful functions of microglia, and the presence of blood-brain barrier, which restricts access of large molecules - including proteins and phospholipids - to microglial targets.

\section{References}

1. Martins RN, Villemagne V, Sohrabi HR, Chatterjee P, Shah TM, et al. (2018) Alzheimer's disease: A journey from amyloid peptides and oxidative stress, to biomarker technologies and disease prevention strategies-gains from AIBL and DIAN cohort studies. J Alzheimers Dis 62: 965-992. [Crossref]

2. Morris GP, Clark IA, Vissel B (2018) Questions concerning the role of amyloid-beta in the definition, aetiology and diagnosis of Alzheimer's disease. Acta Neuropathol 136: 663-689.

3. Ginhoux F, Greter M, Leboeuf M, Nandi S, See P, et al. (2010) Fate mapping analysis reveals that adult microglia derive from primitive macrophages. Science 330: 841-845.

4. Gomez Perdiguero E, Klapproth K, Schulz C, Busch K, Azzoni E, et al. (2015) Tissueresident macrophages originate from yolk-sac-derived erythro-myeloid progenitors. Nature 518: 547-551.

5. Watters JJ, Pocock JM (2014) Microglial physiology. In "Microglia in Health and Disease". Tremblay ME, Sierra A (Eds) Springer-Verlag, New York, NY, USA, pp. 47-80.

6. Wolf SA, Boddeke HW, Kettenmann H (2017) Microglia in physiology and disease. Annu Rev Physiol 79: 619-643.

7. Rymo SF, Gerhardt H, Wolfhagen Sand F, Lang R, Uv A, Betsholtz C (2011) A two-way communication between microglial cells and angiogenic sprouts regulates angiogenesis in aortic ring cultures. PLoS One 6: e15846.

8. Reemst K, Noctor SC, Lucassen PJ, Hol EM (2016) The indispensable roles of microglia and astrocytes during brain development. Front Hum Neurosci 10: 566.

9. Wu Y, Dissing-Olesen L, MacVicar BA, Stevens B (2015) Microglia: Dynamic mediators of synapse development and plasticity. Trends Immunol 36: 605-613.

10. ElAli A, Rivest S (2016) Microglia in Alzheimer's disease: A multifaceted relationship. Brain Behav Immun 55: 138-150.

11. Nimmerjahn A, Kirchhoff F, Helmchen F (2005) Resting microglial cells are highly dynamic surveillants of brain parenchyma in vivo. Science 308: 1314-1318.

12. Sierra A, Beccari S, Diaz-Aparicio I, Encinas JM, Comeau S, Tremblay ME (2014) Surveillance, phagocytosis, and inflammation: How never-resting microglia influence adult hippocampal neurogenesis. Neural Plast 2014: 610343.

13. McGeer PL, Itagaki S, McGeer EG (1988) Expression of the histocompatibility glycoprotein HLA-DR in neurological disease. Acta Neuropathol 76: 550-557.

14. McGeer PL, Itagaki S, Tago H, McGeer EG (1987) Reactive microglia in patients with senile dementia of the Alzheimer type are positive for the histocompatibility glycoprotein HLA-DR. Neurosci Lett 79: 195-200.

15. Fakhoury M (2018) Microglia and astrocytes in Alzheimer's disease: Implications for therapy. Curr Neuropharmacol 16: 508-518.

16. Akiyama H, Barger S, Barnum S, Bradt B, Bauer J, et al. (2000) Inflammation and Alzheimer's disease. Neurobiol Aging 21: 383-421.

17. McGeer PL, Schulzer M, McGeer EG (1996) Arthritis and anti-inflammatory agents as possible protective factors for Alzheimer's disease: a review of 17 epidemiologic studies. Neurology 47: 425-432.

18. Cagnin A, Brooks DJ, Kennedy AM, Gunn RN, Myers R, et al. (2001) In-vivo measurement of activated microglia in dementia. Lancet 358: 461-467.

19. Schwab C, Klegeris A, McGeer PL (2010) Inflammation in transgenic mouse models of neurodegenerative disorders. Biochim Biophys Acta 1802: 889-902.

20. Zhou Y, Song WM, Andhey PS, Swain A, Levy T, et al. (2020) Human and mouse single-nucleus transcriptomics reveal TREM2-dependent and TREM2-independent cellular responses in Alzheimer's disease. Nat Med 26: 131-142. [Crossref]

21. Efthymiou AG, Goate AM (2017) Late onset Alzheimer's disease genetics implicates microglial pathways in disease risk. Mol Neurodegener 12: 43 . 
22. Takatori S, Wang W, Iguchi A, Tomita T (2019) Genetic risk factors for Alzheimer disease: Emerging roles of microglia in disease pathomechanisms. Adv Exp Med Biol 1118: 83-116.

23. Martinez FO, Gordon S, Locati M, Mantovani A (2006) Transcriptional profiling of the human monocyte-to-macrophage differentiation and polarization: new molecules and patterns of gene expression. J Immunol 177: 7303-7311.

24. Colton CA (2009) Heterogeneity of microglial activation in the innate immune response in the brain. J Neuroimmune Pharmacol 4: 399-418.

25. Edison P, Archer HA, Gerhard A, Hinz R, Pavese N, et al. (2008) Microglia, amyloid, and cognition in Alzheimer's disease: An [11C](R)PK11195-PET and [11C]PIB-PET study. Neurobiol Dis 32: 412-419.

26. McGeer PL, Rogers J, McGeer EG (1994) Neuroimmune mechanisms in Alzheimer disease pathogenesis. Alzheimer Dis Assoc Disord 8: 149-158.

27. Sastre M, Klockgether T, Heneka MT (2006) Contribution of inflammatory processes to Alzheimer's disease: Molecular mechanisms. Int J Dev Neurosci 24: 167-176.

28. Malm TM, Jay TR, Landreth GE (2015) The evolving biology of microglia in Alzheimer's disease. Neurotherapeutics 12: 81-93.

29. Michelucci A, Heurtaux T, Grandbarbe L, Morga E, Heuschling P (2009) Characterization of the microglial phenotype under specific pro-inflammatory and anti-inflammatory conditions: Effects of oligomeric and fibrillar amyloid-beta. Neuroimmunol 210: 3-12.

30. Yu Y, Ye RD (2015) Microglial Abeta receptors in Alzheimer's disease. Cell Mol Neurobiol 35: 71-83.

31. Wenzel TJ, Klegeris A (2018) Novel multi-target directed ligand-based strategies for reducing neuroinflammation in Alzheimer's disease. Life Sci 207: 314-322.

32. Andersson U, Yang H, Harris H (2018) High-mobility group box 1 protein (HMGB1) operates as an alarmin outside as well as inside cells. Semin Immunol 38: 40-48.

33. Bianchi ME (2009) HMGB1 loves company. J Leukoc Biol 86: 573-576.

34. Stros M (2010) HMGB proteins: Interactions with DNA and chromatin. Biochim Biophys Acta 1799: 101-113.

35. Bajwa E, Pointer CB, Klegeris A (2019) The role of mitochondrial damage-associated molecular patterns in chronic neuroinflammation. Mediators Inflamm 2019: 4050796.

36. Gouveia A, Bajwa E, Klegeris A (2017) Extracellular cytochrome c as an intercellular signaling molecule regulating microglial functions. Biochim Biophys Acta Gen Subj 1861: 2274-2281.

37. Schindler SM, Frank MG, Annis JL, Maier SF, Klegeris A (2018) Pattern recognition receptors mediate pro-inflammatory effects of extracellular mitochondrial transcription factor A (TFAM). Mol Cell Neurosci 89: 71-79.

38. Hanisch UK (2013) Proteins in microglial activation--inputs and outputs by subsets Curr Protein Pept Sci 14: 3-15. [Crossref]

39. Leitner GR, Wenzel TJ, Marshall N, Gates EJ, Klegeris A (2019) Targeting toll-like receptor 4 to modulate neuroinflammation in central nervous system disorders. Expert Opin Ther Targets 23: 865-882.

40. Venegas C, Heneka, MT (2017) Danger-associated molecular patterns in Alzheimer's disease. J Leukoc Biol 101: 87-98.

41. Brown GC, Neher JJ (2014) Microglial phagocytosis of live neurons. Nat Rev Neurosci 15: 209-216.

42. Spangenberg EE, Green KN (2017) Inflammation in Alzheimer's disease: Lessons learned from microglia-depletion models. Brain Behav Immun 61: 1-11.

43. Brown GC, Vilalta A (2015) How microglia kill neurons. Brain Res 1628: 288-297.
44. Czeh M, Gressens P, Kaindl AM (2011) The yin and yang of microglia. Dev Neurosci 33: 199-209.

45. Dore E, Boilard E (2019) Roles of secreted phospholipase A2 group IIA in inflammation and host defense. Biochim Biophys Acta Mol Cell Biol Lipids 1864: 789-802.

46. Klegeris A, Li J, Bammler TK, Jin J, Zhu D, et al. (2008) Prolyl endopeptidase is revealed following SILAC analysis to be a novel mediator of human microglial and THP-1 cell neurotoxicity. Glia 56: 675-685. [Crossref]

47. Lowry JR, Klegeris A (2018) Emerging roles of microglial cathepsins in neurodegenerative disease. Brain Res Bull 139: 144-156.

48. Madeira JM, Little JP, Klegeris A (2012) Microglia secretome: From neurotoxins to neurotrophins. In "Microglia: Biology, Functions and Roles in Disease". Kaur C, EngAng L (Eds.) Nova Publishers, New York, NY, USA, pp. 73-91.

49. Orihuela R, McPherson CA, Harry GJ (2016) Microglial M1/M2 polarization and metabolic states. Br J Pharmacol 173: 649-665.

50. Liddelow SA, Guttenplan KA, Clarke LE, Bennett FC, Bohlen CJ, et al (2017) Neurotoxic reactive astrocytes are induced by activated microglia. Nature 541: 481487.

51. Walker DG, Lue LF (2015) Immune phenotypes of microglia in human neurodegenerative disease: Challenges to detecting microglial polarization in human brains. Alzheimers Res Ther 7: 56. [Crossref]

52. Tang Y, Le W (2016) Differential roles of M1 and M2 microglia in neurodegenerative diseases. Mol Neurobiol 53: 1181-1194.

53. Prinz M, Jung S, Priller J (2019) Microglia biology: One century of evolving concepts Cell 179: 292-311.

54. Keren-Shaul H, Spinrad A, Weiner A, Matcovitch-Natan O, Dvir-Szternfeld R, et al. (2017) A unique microglia type associated with restricting development of Alzheimer's disease. Cell 169: 1276-1290 e17.

55. Jimenez S, Baglietto-Vargas D, Caballero C, Moreno-Gonzalez I, Torres M, et al (2008) Inflammatory response in the hippocampus of PS1M146L/APP751SL mouse model of Alzheimer's disease: age-dependent switch in the microglial phenotype from alternative to classic. $J$ Neurosci 28 : 11650-11661.

56. Dionisio-Santos DA, Olschowka JA, O'Banion MK (2019) Exploiting microglial and peripheral immune cell crosstalk to treat Alzheimer's disease. J Neuroinflammation 16 74.

57. Shields AM, Panayi GS, Corrigall VM (2011) Resolution-associated molecular pattern (RAMP): RAMParts defending immunological homeostasis? Clin Exp Immunol 165 292-300.

58. Starossom SC, Mascanfroni ID, Imitola J, Cao L, Raddassi K, et al. (2012) Galectindeactivates classically activated microglia and protects from inflammation-induced neurodegeneration. Immunity 37: 249-263.

59. Kakimura J, Kitamura Y, Taniguchi T, Shimohama S, Gebicke-Haerter PJ (2001) Bip/ GRP78-induced production of cytokines and uptake of amyloid-beta(1-42) peptide in microglia. Biochem Biophys Res Commun 281: 6-10.

60. Pointer CB, Wenzel TJ, Klegeris A (2019) Extracellular cardiolipin regulates select immune functions of microglia and microglia-like cells. Brain Res Bull 146: 153-163.

61. Toth ME, Szegedi V, Varga E, Juhasz G, Horvath J, et al. (2013) Overexpression of Hsp27 ameliorates symptoms of Alzheimer's disease in APP/PS1 mice. Cell Stress Chaperones 18: 759-771.

62. Ordonez-Gutierrez L, Re F, Bereczki E, Ioja E, Gregori M, et al. (2015) Repeated intraperitoneal injections of liposomes containing phosphatidic acid and cardiolipin reduce amyloid-beta levels in APP/PS1 transgenic mice. Nanomedicine 11: 421-430. [Crossref]

Copyright: (C2020 Klegeris A. This is an open-access article distributed under the terms of the Creative Commons Attribution License, which permits unrestricted use, distribution, and reproduction in any medium, provided the original author and source are credited. 